\title{
GEMEINNÜTZIGKEIT
}

\section{Neue steuerliche Aspekte bei der Betriebsaufspaltung}

\author{
Siegfried Rutz und Andreas Vollmer
}

Eine neue Betrachtungsweise scheint sich bei der Betriebsaufspaltung eines Zweckbetriebes durchzusetzen: Die Vermietung oder Verpachtung der wesentlichen Betriebsgrundlagen durch die gemeinnützige Besitz-Körperschaft an die gemeinnützige Betriebs-Körperschaft ist keine steuerfreie Vermögensverwaltung, sondern originäre gemeinnützige Tätigkeit.

Gemeinnützige Körperschaften verlagern bei der Ausgliederung eines Zweckbetriebes in der Regel nur denjenigen Betriebsteil, der zur Durchführung der gemeinnützigen und mildtätigen Leistung erforderlich ist. Denn schon allein aus dem Grund, die Grunderwerbsteuer zu sparen, wird die wesentliche Betriebsgrundlage, die aus dem Gebäude besteht, nicht ausgegliedert. Nach herrschender Meinung führt diese übliche Vorgehensweise bei einer sogenannten Betriebsaufspaltung dazu, dass das bei der Besitz-Körperschaft verbleibende Gebäude an die (abgespaltene) Betriebs-Körperschaft vermietet oder verpachtet wird. Die daraus resultierenden Einnahmen stellen bei der Besitz-Körperschaft Einnahmen in der steuerlichen Sphäre der Vermögensverwaltung dar.

Von der herrschenden Meinung wurde diese Sphärenverschiebung des Zweckbetriebsgebäudes in das Vermögensverwaltungsgebäude - ohne dass sich an der Durchführung der Zweckverwirklichung in dem Gebäude etwas ändert - bisher nicht in Frage gestellt.

Die Autoren begrüßen es, dass nun doch eine andere Sichtweise durch einen Fachmann des gemeinnützigen
Steuerrechts begründet wurde und stellen im Folgenden den Kern seiner Aussagen vor: Die Vermietung oder Verpachtung des Gebäudes stellt keine Vermögensverwaltung dar, sondern originäre gemeinnützige Tätigkeit. Eine Verschiebung der steuerlichen Sphären findet nicht statt. Dieser Ansicht ist zu wünschen, dass sie sich bald zu einer herrschenden Meinung in der Literatur und einer gesicherten Praxis mausert.

\section{Ausgangspunkt: Urteil des Bun- desfinanzhofes}

In einem Beitrag für die Zeitschrift »Der Betrieb« (Heft 31 vom 3. August 2007) setzt sich Regierungsdirektor a. D. Werner F. Jost mit den Auswirkungen des Urteils des Bundesfinanzhofes (BFH) vom 29. März 2006 - X R 59/00 (erhältlich unter http://lexetius.com/2006,1078) auf Fälle der Betriebsaufspaltung im steuerfreien Bereich gemeinnütziger Körperschaften auseinander. In diesem Urteil entschied das Gericht, die Gewerbesteuerbefreiung nach $\S 3$ Nr. 20 Buchstabe c GewStG gelte bei einer Betriebsaufspaltung auch für die Vermietungs- und Verpachtungstätigkeit des Besitzpersonenunternehmens.

Jost untersucht, ob die Grundsätze dieses BFH-Urteils auch für Betriebsaufspaltungsfälle bei nach $\S 5$ Abs. 1 Nr. 9 KStG, § 3 Nr. 6 GewStG befreite Körperschaften gelten. Der Bundesfinanzhof hat das inzwischen selbst im Urteil vom 19. Oktober 2006 - IV R 22/02 (erhältlich unter http://lexetius.com/2006,2964 ) bejaht. Es lohnt sich, die Argumentation von Jost im Einzelnen nachzuvollziehen, weil sie - wenn sie herrschende Meinung würde - erhebliche Auswirkungen auf

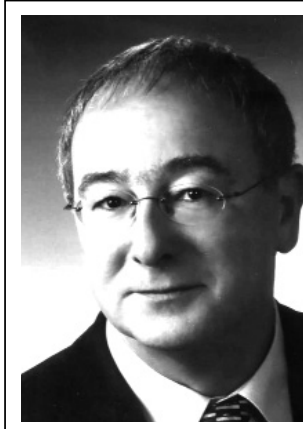

Siegfried Rutz ist in Berlin selbstständiger Unternehmensberater für gemeinnützige

Betriebe. Er ist auch als Dozent für betriebswirtschaftliche Themen bei der Paritätischen Bundesakademie und der Bundesakademie der Arbeiterwohlfahrt tätig.

Internet http://www.beratungrutz.de

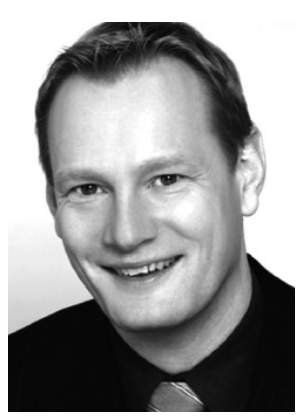

DiplomKaufmann Andreas Vollmer ist in Berlin als selbstständiger Wirtschaftsprüfer und Steuerberater, u. a. mit dem Arbeitsschwerpunkt gemeinnützige Körperschaften tätig. Er ist Dozent an der Paritätischen Bundesakademie und an der Bundesakademie der Arbeiterwohlfahrt sowie Mitglied im Institut der Wirtschaftsprüfer $e$. $V$.

Internet http://www.wp-vollmer.de

die steuerliche Beurteilung der Betriebsaufspaltungspraxis der gemeinnützigen Körperschaften hätte.

Anlass für das Urteil der Münchner Richter vom 29. März 2006 war folgender Sachverhalt: Eine $\mathrm{GmbH}$ betreibt auf einem gepachteten Grundbesitz ein psychiatrisches Wohn- und Pflegeheim. Sie erfüllt die Voraussetzungen der Steuerbefreiung nach § 3 Nr. 20 Buchstabe c GewStG. Der Verpächter sei - so die Auffassung des beklagten Finanzamtes - nicht deshalb von der Gewerbesteuer befreit, weil die Wohnund Pflegeheim-GmbH (Betriebsgesellschaft) die Voraussetzungen der Steuerbefreiung erfüllt habe.

Der Bundesfinanzhof urteilte jedoch, dass die Gewerbesteuerbefrei- 
ung nach § 3 Nr. 20 Buchstabe c GewStG nicht nur dem Betriebsunternehmen, sondern auch dem Besitzunternehmen zugutekommt. Die (isoliert betrachtet) vermögensverwaltende Tätigkeit des Besitzunternehmens wandelt sich im Wege der »Abfärbung « in eine gewerbliche Tätigkeit, die ebenfalls steuerbefreit ist. Entscheidend für den Bundesfinanzhof war die folgende Überlegung: Wenn beim Besitzunternehmen - vor der Betriebsaufspaltung eine Steuerpflicht gar nicht besteht, kann eine solche auch nicht umgangen werden. Die mit § 3 Nr. 20 GewStG steuerbefreiten Zwecke (wie Altenheime, Pflegeheime usw.) würden deshalb im Fall der Betriebsaufspaltung entgegen den Absichten des Gesetzgebers nur unvollkommen erreicht, wenn die Erstreckung des Befreiungstatbestandes auf das Besitzunternehmen verneint würde.

Jost behandelt die Rechtsfolgen des BFH-Urteils hinsichtlich der drei möglichen Varianten der Betriebsaufspaltung im Bereich gemeinnütziger Körperschaften: Betriebsaufspaltung von Zweckbetrieben, Betriebsaufspaltung von »Hilfstätigkeiten «, Betriebsaufspaltung von Vermögensverwaltungsbereichen.

\section{Betriebsaufspaltung von Zweckbetrieben}

Bei der Betriebsaufspaltung eines Zweckbetriebes (zum Beispiel: Abspaltung des Betriebs der Pflegeeinrichtung durch die gGmbH vom Gebäude der Pflegeeinrichtung, das im Besitz des gemeinnützigen e. V. verbleibt) ist es herrschende Meinung, dass die Vermietung und Verpachtung der wesentlichen Betriebsgrundlagen (beispielsweise Gebäude) des bisherigen eigenen Zweckbetriebs an die gemeinnützige Betriebs-Körperschaft steuerfreie Vermögensverwaltung der Besitz-Körperschaft darstellt. Die bei der Besitz-Körperschaft verbliebenen wesentlichen Betriebsgrundlagen wechseln aus der Sphäre des Zweckbetriebes in die Sphäre der Vermögensverwaltung.

Die Anwendung des BFH-Urteils jedoch hat nach Auffassung von Jost zur Folge, »dass die Vermietung/Verpachtung der wesentlichen Betriebsgrundlagen an die ihrerseits gemeinnützige Betriebs-GmbH bei der gemeinnützigen Besitz-Körperschaft nicht mehr nur als zwar ebenfalls steuerfreie Vermögensverwaltung anzusehen ist, sondern als originäre gemeinnützige Tätigkeit - z. B. Zweckbetrieb Krankenhausvermietung an Betriebs-GmbH - zu beurteilen ist. Nur dadurch kommt es zu einer völligen rechtlichen Gleichstellung mit dem Betriebsaufspaltungsfall des § 3 Nr. 20 GewStG, bei dem die Tätigkeit des Besitzunternehmens ebenfalls originär unter diese Steuerbefreiung fällt.«

Die Behandlung als Zweckbetrieb hat Folgen:

- Die gemeinnützige Besitz-Körperschaft muss neben dem Zweckbetrieb Vermietung und Verpachtung im Gegensatz zur bisherigen Rechtslage keine weitere eigene gemeinnützige Tätigkeit mehr ausüben.

- Weil die Überlassung der wesentlichen Betriebsgrundlagen einen Zweckbetrieb darstellt, entfällt die Prüfung, ob die Vermögensverwaltung der gemeinnützigen BesitzKörperschaft gegebenenfalls das Gepräge gibt und damit der gemeinnützige Status der Besitz-Körperschaft gefährdet wäre.

- Die Finanzierung der Beteiligung aus steuerbegünstigten Mitteln der Besitz-Körperschaft ist unbedenklich, da sie keinen Verstoß gegen $\S$ 55 Abs. 1 Nr. 1 und Nr. 5 darstellt.

\section{Betriebsaufspaltung von Hilfstätigkeiten}

In solchen Fällen liegt der Sachverhalt vor, dass die gemeinnützige Besitz-Körperschaft weiterhin die steuerbegünstigte Tätigkeit ausübt (beispielsweise Betrieb einer Pflegeeinrichtung), während die BetriebsKörperschaft diverse Hilfstätigkeiten übernimmt, die durch Betriebsaufspaltung auf sie ausgelagert wurden (z. B. Reinigung, Hausmeistertätigkeiten, Verwaltungstätigkeiten). Nach derzeitiger Rechtslage und herrschender Meinung ist die Übernahme von Hilfstätigkeiten durch die BetriebsKörperschaft steuerpflichtig. Auch in solchen Fällen sieht Jost jedoch eine Abfärbung im Sinne des BFH-Urteils vom 29. März 2006 mit den Folgen, dass
- die Betriebs-Körperschaft ebenfalls nach § 5 Abs. 1 Nr. 9 KStG, § 3 Nr. 6 GewStG steuerfrei wäre;

- bei der Besitz-Körperschaft ein Zweckbetrieb entsteht, der als »Besitzunternehmen für die Hilfstätigkeiten-Körperschaft « bezeichnet werden kann;

- die Prüfung entfällt, ob der steuerpflichtige wirtschaftliche Geschäftsbetrieb (nicht wie Jost hier irrtümlich annimmt die Vermögensverwaltung) der gemeinnützigen Besitz-Körperschaft gegebenenfalls das Gepräge gibt und damit der gemeinnützige Status der Besitz-Körperschaft gefährdet wäre;

- die Finanzierung der Beteiligung aus steuerbegünstigten Mitteln der Besitz-Körperschaft unbedenklich ist, da sie keinen Verstoß gegen $\S$ 55 Abs. 1 Nr. 1 und Nr. 5 darstellt.

\section{Betriebsaufspaltung von Vermögensverwaltungsbereichen}

Auch für den Fall, dass durch eine Betriebsaufspaltung eine BetriebsKörperschaft die bisher von der gemeinnützigen Besitz-Körperschaft selbst betriebene Vermögensverwaltung übernimmt, sieht Jost die Steuerbefreiung im Sinne des BFH-Urteils vom 29. März 2006 für gegeben. Um letzte Zweifel zu beseitigen, wäre eine entsprechende eigenständige Befreiungsvorschrift für Vermögensverwaltungsgesellschaften von gemeinnützigen Körperschaften empfehlenswert.

Maßgebliche rechtliche Grundlage für die Überlegungen von Jost, dem uneingeschränkt zuzustimmen ist, ist für alle Varianten der Betriebsaufspaltung im Bereich der steuerbefreiten Körperschaften der Rechtscharakter des $\S 5$ Abs. 1 Nr. 9 KStG und des $§ 3$ Nr. 6 GewStG als Sozialzwecknorm, womit dem Willen des Gesetzgebers nach der Steuerbegünstigung sozialer Zwecktätigkeiten Rechnung zu tragen ist.

Der Beitrag von Jost kann durch die Bestellung des Einzelheftes 31/2007 „Der Betrieb « (7,50 Euro zuzüglich Versandkosten) unter http://www.der-betrieb.de bezogen werden. Es wäre auch interessant, sich zu vergewissern, dass der Beitrag beim Steuerberater bekannt ist. 